\title{
Contribution of the unfolded protein response to breast and prostate tissue homeostasis and its significance to cancer endocrine response
}

\author{
Inês Direito, Margarida Fardilha* and Luisa A. Helguero*, \&
}

Institute for Biomedicine (iBiMED), Department of Medical Sciences, Universidade de Aveiro

\author{
"Senior authors \\ \& corresponding author: \\ Hormones \& Cancer Lab - Institute for Biomedicine \\ Department of Medical Sciences, University of Aveiro \\ E-Mail: luisa.helguero@ua.pt
}

Tel: +35 1234247 240; ext 22112

Abbreviations: 4OH-TAM, 4-hydroxytamoxifen; AR, androgen receptor; ATF4, activating transcription factor 4; ATF6, activating transcription factor 6; $\mathrm{BC}$, breast cancer; $\mathrm{BiP}$, sensor-binding immunoglobulin protein; CHOP, C/EBP homologous protein; DHT, dihydrotestosterone; E2, 17ß-estradiol; elF2 $\alpha$, eukaryotic initiation factor 2a; EnR, endoplasmic reticulum; ERa, estrogen receptor alpha; ERG, ETS related gene; $I P_{3}$, inositol triphosphate; IRE1 $\alpha$, inositol-requiring enzyme $1 \alpha ; m T O R$, mammalian target of rapamycin; PC, prostate cancer; PERK, protein kinase RNA-like endoplasmic reticulum kinase; PLCy, phospholipase C gamma; PQC, protein quality control; TAM, hydroxytamoxifen; UPR, unfolded protein response; UPS, ubiquitinproteasome-system; XBP-1, X-box binding protein 1; XBP-1s, spliced XBP-1; XBP-1u, unspliced XBP-1. 


\section{Abstract}

Resistant Breast and Prostate cancer remain a major clinical problem and new therapeutic approaches and better predictors of therapeutic response are clearly needed. Due to the involvement of the Unfolded Protein Response (UPR) in cell proliferation and apoptosis evasion, an increasing number of publications support the hypothesis that impairments in this network trigger and/or exacerbate cancer. Moreover, UPR activation could contribute to the development of drug-resistance phenotypes in both breast and prostate cancers. Therefore, targeting this pathway has recently emerged as a promising strategy in anti-cancer therapy. This review addresses the contribution of UPR to breast and prostate tissues homeostasis and its significance to cancer endocrine response with focus on the current progress on UPR research related to cancer biology, detection, prognosis and treatment are also discussed. 


\section{Introduction}

Breast cancer $(\mathrm{BC})$ and prostate cancer $(\mathrm{PC})$ are currently the first and fourth cause of death among female and male cancer patients worldwide (1). About $70 \%$ of all diagnosed BCs express estrogen receptor alpha (ERa) and $90 \%$ of all PCs express androgen receptor $(A R)$. ERa and $A R$ are ligand-activated transcription factors which exert their molecular functions by regulating gene expression through genomic and non-genomic mechanisms. These two proteins are still to date the gold standard for diagnosis and treatment decisions. Despite the favorable improvements in patients' survival associated with endocrine therapies, about $50 \%$ initially hormone responsive BCs (2) and almost all PCs $(3,4)$ develop resistance to these treatments. This contributes to the progression of the disease towards an incurable and lethal stage. Therefore, understanding the molecular mechanisms involved in intrinsic or acquired resistance remains a major clinical challenge.

Several studies have pointed out ERa and AR cross-talk with receptor tyrosinekinase pathways as a key alteration leading to endocrine resistance (5-10). Moreover, receptor activating mutations are also associated to endocrine resistance in about 15$20 \%$ BC (11) and 10\% PC (12), respectively. Recent evidence also suggests that adaptive stress responses which have evolved to protect cells from proteotoxic stress may play a role in endocrine resistance (13-16).

Adaptive stress responses are activated in response to metabolic and environmental stress and involve the protein quality control (PQC) network. The PQC comprises the translational machinery, protein degradation machinery as well as molecular chaperones and co-chaperones (17). Normal cells respond to acute stress by activating the PQC in order to rapidly restore protein homeostasis (from now on proteostasis) and thus protect from proteotoxic stress. However, if proteostasis is not restored cells commit to apoptosis. Therefore, the PQC network targets survival or 
death pathways, which are selectively activated depending on the restoration capacity of the cells.

An increasing number of publications support the hypothesis that impairments in PQC mechanisms trigger and/or exacerbate cancer, with some components of these network associated to therapy resistance in both $\mathrm{BC}$ and $\mathrm{PC}(15,18-25)$. In this review, we discuss the current knowledge on the deregulation of one of these PQC mechanisms, the unfolded protein response (UPR), in breast and prostate tissue homeostasis, in BC and PC, as well as its significance to endocrine therapy resistance.

\section{The Unfolded Protein Response}

This pathway is activated to protect cells from detrimental conditions that activate endoplasmic reticulum (EnR) stress. As such, hypoxia, acidosis, nutrient deprivation and gene mutations that contribute to protein misfolding cause EnR stress leading to UPR activation (26) followed by restoration of EnR functions (protein folding, post-translational modifications, lipid and steroid synthesis and calcium signaling) (27). UPR is initiated when the chaperone EnR stressor sensor-binding immunoglobulin protein $(\mathrm{BiP})$ frees itself from the EnR-resident proteins that regulate the UPR cascades and in turn associates with misfolded proteins. This results in activation of

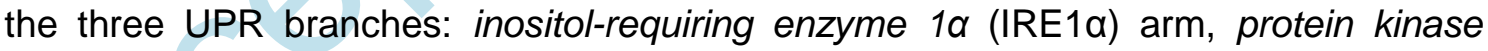
RNA-like endoplasmic reticulum kinase (PERK) arm and activating transcription factor

6 (ATF6) arm (Figure 1). UPR activation results in two temporally distinct events to allow EnR recovery: an initial reaction to decrease mRNA transcription, lower protein translation rates and enhance degradation of damaged proteins, followed by a later induction of genes involved in proteostasis and lipid biosynthesis control. Therefore, cells can adapt to different intensity and duration of intrinsic and environmental stress by selectively regulating UPR branch activation (26-28). 
IRE1 1 branch: Upon dissociation from BiP, IRE1 dimerizes and autophosphorylates activating its RNAse domain, leading to the subsequent splicing of $X$-box binding protein 1 (XBP-1) mRNA. Spliced XBP-1 (XBP-1s) encodes a transcription factor that up-regulates genes involved in protein folding, lipid metabolism, quality control and endoplasmic-reticulum-associated degradation (ERAD). XBP-1 heterodimerizes with several other transcription factors, hence its target genes may vary according to the cell context and stimuli. Unspliced XBP-1 (XBP-1u) can function as a negative regulator of $\mathrm{XBP}-1 \mathrm{~s}$ activity, therefore $\mathrm{XBP}-1 \mathrm{~s} / \mathrm{XBP}-1 \mathrm{u}$ balance can have significant consequences for UPR activation as well as XBP-1 function and transcription of its target genes $(29,30)$. IRE1 $\alpha$ also regulates the stability of multiple RNAs through its endonuclease activity in a process known as regulated IRE1-dependent decay (RIDD) which targets glucose metabolism, inflammation and apoptosis. IRE1a cleavage of XBP-1 or activation of RIDD follow different kinetics and may depend on IRE1 $\alpha$ oligomeric state (31).

PERK branch: Release of PERK from BiP results in PERK oligomerization and transautophosphorylation, which activates its kinase function. p-PERK phosphorylates eukaryotic initiation factor $2 \alpha$ (elF2 $\alpha$ ) in Ser51 to reduce the rate of formation of the elF2 ternary complex (elF2-GTP-tRNA Methionine) which is essential for ribosome binding to the start codon (32). Despite this translational inhibition, some mRNAs, such as activating transcription factor 4 (ATF4), escape and are translated (33). ATF4 induces the expression of anti-oxidative enzymes, promotes amino acid synthesis, autophagy, protein folding and differentiation, and downregulates genes involved in cellular senescence and inhibitors of angiogenesis $(27,34)$. In response to chronic stress, sustained ATF4 expression induces C/EBP homologous protein (CHOP) gene transcription, which encodes a transcription factor that stimulates growth arrest and apoptosis (35). CHOP's target gene, growth arrest and DNA damage-inducible protein 
34 (GADD34) in association with phosphatase protein 1 (PP1) dephosphorylate elF2a, which enables the recovery of protein translation (36). However, if CHOP accumulates, due to chronic stress, Bcl-2-like protein 11 (BIM) expression is induced, and cells commit to apoptosis (35). Thus, PERK arm integrates adaptive and chronic EnR stress responses.

ATF6a branch: Following its release from BiP, ATF6a translocates to the Golgi where it is cleaved by the proteases SP1 and SP2, releasing an N-terminal fragment (ATF6f) that acts as a transcription factor of XBP-1u, EnR chaperones including BiP and ERAD-associated proteins $(26,27,37)$. ATF6f also forms heterodimers with XBP-1s which drives specific gene expression programs. Despite the functional overlap between ATF6 $\alpha$ and IRE1 $\alpha$ branch gene targets, it appears that ATF6ahas evolved to enhance the protective mechanisms of PERK and IRE1 $\alpha$ signaling, contributing for cell survival during chronic stress (38). In fact, ATF6a deletion results in impaired function of the secretory pathway during EnR stress thus resulting in impaired long-term EnR function (39).

\section{Role of the unfolded protein response in tissue homeostasis: focus on the} mammary gland

During lactation, normal breast cells must balance the increased production of milk proteins with the risk that an excessive protein load accumulation could impair basic cell survival functions $(28,37,40)$. Similarly, to observations in other secretory cells such as plasmocytes and pancreatic cells, mammary cells have a well-coordinated and active UPR to adapt to the high EnR activity required during their functional differentiation during pregnancy and lactation (41-45). Notably, to the date, there is no information available regarding UPR involvement in normal prostate physiology and development. 
IRE1-a/XBP1 branch: A thorough characterization of mammary tissue from virgin, early pregnant and lactating mice lacking XBP-1 disclosed that its deletion correlates to poor branching morphogenesis and impaired terminal end bud formation at the virgin stage, possibly due to a stromal effect. XBP-1 deletion was sufficient to impair lobuloalveolar development during early lactation. This is due to reduced epithelial cell proliferation which prevents lobuloalveolar compartment expansion as shown using tissue transplantation techniques (41). The same authors also showed that XBP-1 was detectable only during lactation and was nearly absent in virgin and pregnant mice. However, other have shown that XBP-1mRNA and protein gradually increase from pregnancy, reaching highest levels during lactation. Moreover, in agreement with a role for XBP-1 in functional differentiation, blocking its expression in HC11 mammary epithelial cells reduced lactogenic protein mRNA levels in response to dexamethasoneprolactin-insulin stimulation. The authors explained these as resulting from reduced mRNA of prolactin and insulin receptors in cells with XBP-1 knock-down (42). Therefore, the increased protein and lipid synthesis demands during lactogenic

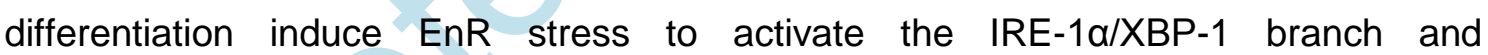
consequently increase the EnR capacity needed to support alveolar expansion and the secretory phenotype.

PERK/ATF4 branch: PERK activation inhibited MCF10A acini formation under normal growth conditions and its inhibition with dominant-negative PERK mutants resulted in hyperproliferation and in vivo tumorigenicity (43). In line with this, overexpression of the PERK downstream effector ATF4 in mice decreased proliferation and differentiation of mammary alveolar epithelium and accelerated involution (44). The effect of this branch on involution appears to result from an interplay with autophagy regulation where elevated BiP and p-elF2a expression (as well as XBPu and autophagy genes) occurred 
in the reversible involution phase $24-48 \mathrm{~h}$ postweaning in mice. Whereas, ATF4 expression was increased in the irreversible involution phase $(72-168 \mathrm{~h})$ and stimulated CHOP expression, which coincided with the expression of apoptosis markers such as active caspases and cleaved PARP (46). This study showed a sequential contribution of UPR and autophagy pathways in the involution process, promoting pro-survival or death signaling during the reversible and irreversible involution phases, respectively (46). During mid-lactation, PERK physiological activation appears to be necessary for the lipogenic maturation of mammary epithelial cells, as demonstrated by the increased levels of p-elF2abetween lactation days 7 and 12 (45), as well as activation of the lipogenic phenotype which characterizes lactogenic differentiation (47). Tissue-specific PERK deletion in the mouse mammary epithelium reduced levels of the lipogenic genes (45). Regarding ATF4, its expression was found differentially regulated during the development of the mammary gland although results are not consistent. One study found that in total tissue lysates ATF4 expression was highest during virgin and pregnancy stages and lowest during lactation (44). The second study reported that ATF4 protein levels gradually increased during pregnancy and reached a significantly higher level on lactation days 4-7 (42). Supporting the latter study, in the HC11 cell line, ATF4 knock-down reduced insulin and glucocorticoid receptor mRNA levels which suggests that ATF4 is necessary to allow lactogenic protein synthesis. Moreover, in the same cell line insulin and prolactin increased $\mathrm{CHOP}$ mRNA and transcriptional activity on the STAT5a-driven beta-casein gene (48). However, CHOP's function in lactogenic differentiation remains to be clarified since in whole mouse mammary tissue CHOP mRNA significantly decreased from day 15 of pregnancy to day 4 of lactation (42), while in another study it transiently peaked at lactation day 5 (48).

In summary, PERK activation may induce a positive feedback loop where ATF4 reduces proliferation and increases the response to lactogenic hormones, which in turn 
enhance CHOP's transcriptional activation. Reduced proliferation in ATF4 overexpressing mammary glands could be related to CHOP-induced G0/G1 arrest (49), thus supporting a role of PERK/ATF4 branch in inhibition of alveolar cell proliferation and stimulation of their functional differentiation.

ATF6 branch: there was no significant change in the expression level of ATF6 during the mammary gland developmental stages (42). However, since ATF6 branch enhances the response to IRE-1 $\alpha$ and PERK activation, more studies are needed to asses ATF6 activation.

\section{The Unfolded Protein Response in Breast and Prostate Cancer}

Cancer cells have increased metabolic demands to sustain biomolecule biosynthesis, survive chronic hypoxia, acidosis and nutrient depletion (50). In addition, accumulation of gene mutations alters protein folding which could increase formation of toxic protein aggregates (51). Cancer cells have evolved PQC mechanisms that allow their survival in normally deleterious conditions, with the overall result of chronically disturbed proteostasis along with enhanced survival (52). One explanation for this phenomenon is that cancer cells display sub-functional death pathways, thus chronic proteostasis loss does not result in cell death, but may be a selective advantage contributing to cancer cell survival (27).

Due to the importance of UPR in morphogenesis and differentiation of the secretory epithelium, the exploitation of UPR by neoplastic breast and prostate cells in order to deal with metabolic and oxidative stress seems predictable. This evolutionary strategy could induce cancer cell dormancy (34) and may be involved in the acquisition of a therapy resistant cancer phenotype. Amongst PQC effectors the ubiquitinproteasome-system (UPS) is frequently up-regulated in cancer cells but its activity per seis not sufficient to maintain cancer cell proteostasis (53). Curiously, BC and PC 
cancer stem cells have lower proteasome activity, which is related with higher metastatic capacity, worse prognosis and therapeutic resistance (54-57). Thus, in breast and prostate cancer stem cells protein folding mechanisms must be overactivated to compensate for the low proteasomal activity. In fact, it was recently demonstrated that disseminated $\mathrm{BC}$ with mesenchymal characteristics have higher level of UPR proteins, namely the chaperones BiP and GRP94, thanMDA-MB-468 and MCF7 cells (58).

A number of studies have shown alterations in BC and PC that support the hypothesis that impairments in UPR appear to trigger and/or exacerbate the disease as well as influence response to anti-cancer therapy $(15,18,24,28,59-67)$. However, the current knowledge on UPR function in PC is still limited and controversial: So, et al found that all the 3 UPR branches are selectively down-regulated in mouse models of prostate tumorigenesis (68); on the other hand, Liu et al found that IRE1 $\alpha$, PERK and ATF6 were increased and significantly associated with Gleason grade, T and M stages, PSA level ad shorter survival (62). The following sections focus on receptor-positive BC and PC and the significance of UPR on endocrine response.

\section{Regulation of UPR branch activation by ER and AR agonists and antagonists}

Estrogens and androgens exert their effects through distinct molecular mechanisms (Figure 2). In the classical nuclear receptor (NR) pathway, ER and AR bind their agonist, dimerize and bind to DNA consensus sequences which results in direct gene regulation. ER and AR may also interact with other transcription factors and regulate gene expression by indirect DNA binding (69-71). In the absence of agonist, these NRs can be activated by phosphorylation through cross-talk with receptor tyrosine kinase signaling which results in ligand-independent regulation of gene expression. On the other hand, a ligand activates a membrane-associated receptor or a receptor located in the cytoplasm, such as phospholipase $C(P L C) / p r o t e i n ~ k i n a s e ~ C$ 
(PKCs), Ras/Raf/MAPK, phosphatidyl inositol 3 kinase (PI3K)/Akt and cAMP/ protein kinase $A(P K A)$, to induce a rapid physiological response without direct gene regulation by ER or AR (69-71). Increasing evidence shows that ER or AR activate or inhibit the UPR branches in a cell context-dependent manner and that this could be associated to endocrine therapy resistance.

BiP: BC and PC cells usually overexpress molecular chaperones such as HSP27, HSP60, HSPA1A, GRP94 and BiP $(24,72,73)$ which aid to restore proteostasis by facilitating protein folding and the pro-survival and cytoprotective response of cancer cells to environmental stress (74).

- $\quad$ BiP in breast cancer: Non-genomic activation of Phospholipase C Gamma (PLCY) by ERa is followed by rapid $\mathrm{Ca}^{2+}$ efflux into the cytosol, causing BiP release from UPR effectors and anticipatory UPR activation of all three arms of the UPR $(66,75)$ which prepares the cells for increased protein and lipid synthesis needed for proliferation. A small ERa biomodulator molecule, BHPI, was recently shown to induce a massive and sustained UPR activation involving non-genomic activation of PLCY by ERa, which initiates a fruitless cycle of EnR $\mathrm{Ca}^{2+}$ depletion and ATP consumption and converts UPR from cytoprotective to cytotoxic (76). ERa abundantly interacts with BiP in the EnR, which probably reflects the need of this chaperone for ERa mediated nongenomic signaling (77). In T47-D cells, pre-exposure to E2 for 8 days elicits a 10-fold increase in the concentration of tunicamycin necessary to induce apoptosis (66). Therefore, cells can exploit the UPR anticipatory response produced by ERa activation as a protective mechanism against chemotherapy

E2 induces BiP transcription in MCF-7 and T47-D cells $(48,66,78)$. In E2dependent MCF-7/WS8 cells and in E2-refractory MCF7-2A cells, E2 also upregulates BiP mRNA. On the contrary, in E2-sensitive/anti-estrogen resistant MCF7-5C cells BiP expression was not sufficiently induced and as a result IRE1 $\alpha / X B P-1$ branch was 
activated leading to up-regulation of pro-apoptotic caspase-4 and BIM (79). Thus, in order to support cell survival under estrogen stimulation, cells must up-regulate BiP levels. In agreement with this, in MCF-7/BUS cells which undergo apoptosis in response to $\mathrm{E} 2$ starvation, $\mathrm{BiP}$ over-expression protected from mitochondria permeabilization and apoptosis, while its knock-down sensitized the cells to E2starvation (80). Therefore, BiP induction seems to prevent apoptosis induced by loss of ER activation.

In normal culture conditions, BiP levels were higher in E2-independent cells that were TAM-resistant or antiestrogen resistant (MCF7-RR and MCF-7/LCC9) when compared to those that remained antiestrogen sensitive (MCF-7/LCC1 and MCF-7) (16). In MCF7-RR and MCF-7/LCC9 cells, anti-estrogen treatment elevated BiP mRNA and protein levels to promote survival through distinct pathways: by integrating prosurvival signaling from Tuberous sclerosis 2 (TSC2) and AMP-activated protein kinase (AMPK) with autophagy and UPR (16); and by inhibiting apoptosis through mTOR activation, anti-apoptotic BCL2 family proteins (80) and by reducing caspase-7 (81-83). Supporting this findings, BiP silencing in these cells induced antiestrogen sensitivity (16). On the contrary, BiP over-expression in antiestrogen sensitive MCF7 and LCC1 cells lead to a reduced proliferation rate even in the absence of antiestrogen treatment. The authors suggested that BiP may be involved in the growth-inhibition process and that this function is lost in resistant cells (16). Therefore, BiP appears to be necessary for the maintenance of a resistance phenotype since its over-expression was related with resistance to E2 starvation-induced apoptosis in MCF7-5C, mimicking hormonal therapy resistance (80) and its down-regulation with shRNAs re-sensitizes antiestrogen resistant cells to anti-estrogen treatment (16).

Novel studies have recently shown that in LCC9 anti-estrogen resistant cells and tumors, reducing BiP with an antisense morpholino diminishes de novo fatty acid synthesis and mitochondrial fatty acid transport through down-regulation of SRFBP1 
transcription factor and its downstream genes SCD1, FASN and CPT1A (84).This results in accumulation of linoleic acid, linolenic acid and arachidonic acid due to reduced mitochondrial beta-oxidation and consequent lipotoxicity. Notably, while only reduction of BiP was necessary to reduce SRFBP1 and increase lipid accumulation, co-stimulation with TAM was needed to increase lipid peroxidation and ROS levels. This suggests that high BiP levels in anti-estrogen resistant cells could reduce dependency on ER signaling for survival. Interestingly, BiP morpholinos activated IRE1a-XBP1 branch, which is known to stimulate the SRFBP1 co-activator C/EBPa, as well as lipogenesis genes (85). Still, lipogenic activation by UPR branches is reduced upon chronic stress (85), which explains the results in LCC9 cells and tumors. Metabolic adaptations in endocrine resistance occur through expression of oncogenes like Myc proto-oncogene protein (MYC) (86) or Early growth response protein 1 (EGR1) $(86,87)$. For example, MYC is expressed at higher levels in LCC9 anti-estrogen resistant cells as compared to sensitive counterparts (86) and in glucose-deprived conditions the increase in BiP selectively actives UPR branches to resolve nutritional stress (86). Thus, this body of work has disclosed BiP as an important hub regulating adaptation to nutrient-deprivation and ER signaling that extends beyond the EnR.

T47-D and MCF-7 BC cells, as well as $\mathrm{BC}$ samples have higher basal BiP levels than non-neoplastic human mammary epithelial cells and normal mammary tissues, respectively $(16,66,72,78,88)$. In vivo studies using a DMBA-induced mouse mammary tumor model that mimics the spectrum of TAM responses seen in patients, showed increased BiP expression in tumors with acquired antiestrogen resistance when compared with tumors showing complete response, or de novo resistance (16). DMBA tumors with acquired antiestrogen resistance up-regulated $\mathrm{BiP}$ in response to antiestrogen treatment, supporting the idea that $\mathrm{BiP}$ up-regulation is an adaptive response of resistant cells to endocrine therapy. 
$\mathrm{BiP}$ has also been detected in the cell surface of BC cells (89-91) and was associated with early stages of the disease, with progesterone receptor expression, highest p53 levels and with good prognosis in ERa-positive tumors (90). On the other hand, in triple negative $\mathrm{BC}$, BiP cell surface expression was related with growth inhibition, apoptosis and reduced anti-BiP antibodies in mouse serum (89). Presently, the functional significance of $\mathrm{BiP}$ in the cell surface is unknown but may be related to non-genomic effects as shown in PC cells (see below).

- $\quad$ BiP in prostate cancer: in PC tissue samples BiP is over-expressed as disease progresses from early to metastatic androgen-independent state $(64,65,92)$. BiP seems to be essential for PC cell survival, to allow cells to resolve EnR overload in response to the AR anabolic signaling in nutrient-deprived conditions (93). In fact, BiP temporal up-regulation can occur independently of EnR stress, and promotes acute adaptation to nutrient starvation by blocking autophagy (93). However, BiP-mediated autophagy was shown to be critical for the development of androgen-resistant PC $(16,93)$. Therefore, it is not surprising that $\mathrm{BiP}$ is also associated with endocrine therapy resistance and its inhibition restored endocrine response in C42B castration resistant cells (15).

PC cells acquire resistance to endocrine therapy by translocating BiP from EnR lumen to the cell membrane (94-96). Although the molecular mechanisms involved in BiP translocation are not completely clear, in PC3 cells, the tumor suppressor Proteinase-activated receptor 4 (PAR-4) $(97,98)$ as well as the co-chaperone Dna Jlike protein 1 are required (99). In addition, lost or altered EnR amino acid retention motif KDEL in BiP C-terminal domain may also enhance its surface expression and this mechanism can be dependent or independent of EnR stress (100). As a cell membrane protein $\mathrm{BiP}$ mediates $\mathrm{PI}-\mathrm{K} / \mathrm{Akt}$ signaling to stimulate proliferation, invasion and therapeutic resistance of PC cells (94-96). Therefore, BiP can be localized in the extracellular surface of late stage PC cells, but not in the surface of normal cells, 
opening a new opportunity for using this protein as a diagnostic biomarker and/ or as a drug target (92).

In summary, in endocrine-sensitive cells, BiP overactivation has an inhibitory effect. Yet, it is upregulated as BC and PC progresses, and is associated with endocrine-resistance. Whether BiP subcellular localization pays a role in promoting endocrine-resistance in both $\mathrm{BC}$ and $\mathrm{PC}$ remains to be elucidated.

\section{$\underline{\text { IRE1a/XBP-1 branch: }}$}

- IRE1 $\quad$ branch in breast cancer: MCF-7 and T47-D cells treated with E2 rapidly and transiently increase XBP-1s levels as early as 2 hours after treatment $(66,78)$. XBP-1s over-expression in these cell lines resulted in increased ERa expression and enhanced ERa transcriptional activity, favoring a hormone-independent growth and anti-estrogen resistance (18). This is probably because ERa:XBP-1 alter the expression of several anti-apoptotic genes, such as Apoptosis regulator Bcl-2 (BCL2) and Bcl-2-like protein $2(\mathrm{BCLW})$ to inhibit apoptosis as well as cell cycle genes to prevent cell cycle arrest in G0/G1 phase $(18,101)$.

In normal culture conditions XBP-1s levels are higher in TAM resistant MCF7RR cell than in their sensitive counterparts (20) and TAM sensitivity of these resistant cells could be re-established after treatment with the inhibitor of IRE1 $\alpha / X B P 1$ activation, STF-083010 (102). Moreover, in endocrine resistant T47-D/ERaY537S mutant cells in which ERa is constitutively activated, XBP-1s was also constitutively high and $\mathrm{BiP}$ as well as p58 $8^{\mathrm{IPK}}$ expression was up-regulated (13), while XBP-1 knockdown in anti-estrogen resistant LCC9 cells re-sensitized cells to antiestrogens (103). On the other hand, in anti-estrogen sensitive MCF-7 and T47-D cells ICI 182780 or $4 \mathrm{OH}-\mathrm{TAM}$ treatment inhibited the E2 mediated splicing of XBP-1 (66). XBP-1s is also able to confer E2-independence and resistance to aromatase inhibitors as well as anti- 
estrogens in both MCF-7 and T47-D cells (18). These findings strongly support the link between IRE1 $\alpha$ activation, XBP-1s increase and anti-estrogen resistance.

$\mathrm{XBP}-1 \mathrm{u}$ can also interact with $\mathrm{ER} \alpha$ and enhance its transcriptional activity. Interestingly, when analyzing ERE-Luc activation in MDA-MB-231 cells transfected with ER $\alpha$ and either XBP-1s or XBP-1u, treatment with the anti-estrogens ICI 182780 or $4 \mathrm{OH}-\mathrm{TAM}$ completely blocked the synergistic effects of XBP-1u on ERa transcriptional activity in the presence or absence of E2, whereas both ICI 182780 and, to a lesser extent, $4 \mathrm{OH}-\mathrm{TAM}$ reduced but did not abolish the ability of XBP-1s to transactivate ERa (101). Thus, an increase in XBP-1s/XBP-1u ratio may indicate a loss of XBP-1u corepressor function in the presence of antiestrogens, leading to endocrine resistance. XBP-1s protein interaction with hypoxia-inducible factor $1 \alpha$ (HIF1 $\alpha$ ) increases cell tolerance to hypoxia, facilitating tumor growth by a mechanism independent of angiogenesis $(104,105)$. HIF-1 $\alpha$ is an ERa direct transcriptional target and both proteins share many target genes. HIF-1 $\alpha$ is able to confer anti-estrogen resistance to MCF-7 cells (106). Therefore, XBP-1s co-activator function can enhance HIF-1a/ERa cross-talk to facilitate endocrine resistance.

The cross-talk between ER and NF-K $\beta$ in endocrine resistance is well documented and has been reviewed elsewhere (107). These interdependencies have a significant impact on cell survival, especially in cells with elevated IKK/ NF-K $\beta$ activity such as breast (108) and prostate cancer cells (107), activating downstream NF-k $\beta$ signaling $(101,103)$. XBP-1 was shown to regulate NF-k $\beta$ signaling. Indeed, antiestrogen resistant cells up-regulate p65/RelA while, XBP-1 inhibition with siRNAs reduced NF-k $\beta$ gene reporter activation through downregulation of RelA/p65 mRNA levels (103). The authors used an ingenious approach to study if XBP-1s or XBP-1u was responsible for these effects and found that while both isoforms can activate NF$k \beta$, XBP-1u needs ERa expression, while XBP-1s can also induce p65/RelA expression independently of ERa signaling. Thus, while both isoforms can influence 
cell fate decisions by affecting the balance between apoptosis and pro-survival autophagy, XBP-1s is more potent in activating NF-K $\beta$ signaling (103). In addition, in combination with translation repression by PERK, IRE1 $\alpha$ was able to maintain IKK basal activity, which is critical for maximal NF-K $\beta$ activation during UPR activation (109).

In opposition to ERa, which promotes $\mathrm{BC}$ cell proliferation, ER $\beta$ can, in certain conditions, counterbalance ERa effects and inhibit proliferation and survival $(110,111)$. ER $\beta$ over-expression in BC cells has been associated with anti-metastatic and antiproliferative responses (112), being its expression correlated with an improved response to endocrine therapy (113) and increased patient survival $(66,114)$. ERß1 isoform in both antiestrogen sensitive and resistant BC cells, activates death pathways through IRE1 $\alpha / X B P-1$ down-regulation while isoform ERß2 does not alter XBP-1s activity nor BC sensitivity to stress inducers (115).

Changes in XBP-1s levels have been associated with poor prognosis and with anti-estrogen resistance $(18,20,30,61,78,101,116,117)$. On the other hand, XBP-1u favors apoptosis via dominant-negative downregulation of XBP-1s in ERa-positive BC cells (30). In fact, a study of $100 \mathrm{BC}$ samples from patients treated with adjuvant TAM showed that a higher XBP-1s/XBP-1u mRNA ratios were associated with grade 3 tumors, higher proliferation index and poor survival in ERa-positive cases as well as ERa- and PR-negative cases (30). XBP-1 mRNA expression has also been shown to predict a poor response to TAM therapy (30).

Taken together, these results point towards activation of IRE1 $\alpha$ to enhance ER $\alpha$ activity and possibly endocrine resistance through up-regulation of XBP-1s which induces transcriptional activation of ER $\alpha$ target genes as well as NFK $\beta$ activation, stimulating both estrogen-dependent and independent cell cycle progression and inhibiting antiestrogen induced apoptosis (28). More studies are needed to understand the interplay between IRE1a/XBP-1s UPR branch and ER $\beta$. However, XBP-1s appear 
as a key co-activator of ERa that is inhibited by ERß1, thus counteracting ERa's positive regulation of cell cycle and pro-survival genes which supports observations where ER $\beta$ over-expression increases endocrine sensitivity $(113,118)$.

- IRE1a/XBP-1s in prostate cancer: in androgen-responsive LNCaP and PC3 cells, AR activation can directly upregulate the expression of IRE1 $\alpha$ branch target genes, including IRE1 $\alpha$ and XBP-1s themselves. This leads to activation of proliferative signaling and simultaneous inhibition of pro-apoptotic JNK signaling; an effect reversed by IRE1a or XBP-1 knock-down (61). In these experiments, IRE1a mRNA and protein increased in a time-dependent manner from 6h until 36h following R1881 treatment, thereafter, levels decayed but remained significantly higher than the control throughout the time-course study of $184 \mathrm{~h}$. XBP-1s mRNA increase was evident after $12 \mathrm{~h}$ and showed the same dynamics as IRE1a. In agreement with this, androgen withdrawal in CWR22 cells induces a significant decrease of IRE1 $\alpha$ and XBP-1s levels by 72 hours, nearing basal levels at 120h, whereas XBP-1u levels were not affected, resulting in increased apoptosis of CWR22 cells and tumor regression (61). It has been reported that XBP-1s mRNA levels do not change in LNCaP cells after 6 hours treatment with the AR agonist Mibolerone (93), which is possibly due to the fact that XBP-1s increase occurs at later time points as discussed above.

In summary, BC and PC cells respond to ERa and AR activation by upregulating the IRE1 $\alpha / X B P-1$ pathway. However, in PC cells, this response does not seem to be non-genomic/anticipatory as observed in BC, but a genomic and sustained effect. In endocrine resistant BC cells antiestrogen treatment possibly increases $\mathrm{ER} \alpha / \mathrm{XBP}-1 \mathrm{~s}$ transcriptional activity, but in sensitive cells this is prevented by increasing the XBP1u/XBP-1s ratio. Currently, there are no studies reporting the effects of antiandrogens on the activation of this UPR branch. 


\section{PERK/elF2 $\alpha$ arm:}

- $\quad$ PERK/elF2 $\alpha$ arm in breast cancer: In T47-D cells, p-PERK levels are increased 15 minutes after E2 stimulation and decrease after 45 minutes. This is accompanied by an increase in p-elF2aand in ATF4, resulting in a modest and transient decline of protein synthesis that is maintained for 40 minutes and without pro-apoptotic CHOP induction. In the same study ICI 182780 blocked the inhibition of protein synthesis by E2 (66). ICl 182780 induces ERa protein aggregation and its degradation by the proteasome (119). Therefore, upon ICI 182780 treatment the cell's clearing mechanism are being used and preventing the anticipatory response to E2 which may result in unresolved proteotoxic stress leading to ICI 182780 sensitivity. In fact, longterm treatment of MCF-7/LCC1 antiestrogen sensitive cells with ICI 182780 (6 days) promotes sustained EnR stress, causing a further activation of apoptotic signaling through PERK-CHOP activation and BCL2 down-regulation (19).

$\mathrm{PISK} / \mathrm{Akt} / \mathrm{mTOR}$ pathway activation is a clinically relevant aspect of ERapositive endocrine resistant BC (120-123). MCF-7 cells with constitutive Akt activation escape from growth inhibition induced by $4 \mathrm{OH}-\mathrm{TAM}$. This mechanism involves both ligand-dependent and independent activation of ERa in part by mTOR signaling pathway activation (124). Akt negatively regulates PERK through phosphorylation at threonine 799, this prevents induction of apoptosis under severe or chronic EnR stress (125) and could be one mechanism used by resistant cells to evade apoptosis. Moreover, PERK ablation impairs NRF-2 antioxidant response triggering DNA damage (126). On the other hand, under oxidative stress conditions, Akt promotes cell death (127) and this can be antagonized by PERK activation (125) and downstream NRF-2regulated transcription of antioxidant enzymes $(128,129)$ which has already been shown to protect BC cells from chemotherapy (130). Since during prolonged EnR stress PERK-elF2 $\alpha$ constitute a switch from pro-survival to pro-apoptotic signaling through ATF4, CHOP and NRF-2 up-regulation (131) and the cross-talk between UPR 
and PI3K/Akt/mTOR pathway regulates cell survival in response to different tumor micro-environment insults, stress resolution seems to be dependent on the cellular context and the hierarchical organization of the PERK and PI3-K pathway components.

NF-k $\beta$ over-expression can confer estrogen-independence and antiestrogen resistance because of an overlap in their target genes (132) and trans-repressive interaction between these two proteins (107). NF-k $\beta$ is a major stress-inducible antiapoptotic transcription factor and elF2ainactivation by PERK inhibits the synthesis of NF-k $\beta$ inhibitor IkB, thereby enhancing NF-kßanti-apoptotic activity in stressed BC cells $(133,134)$. Moreover, NF-k $\beta$ subunit p65 can repress CHOP expression in BC cells, thus protecting cells against EnR stress-induced death $(135,136)$ through the expression of pro-survival genes like BCL-2s, TRAF1/TRAF2 and SOD (137). Therefore, PERK activation could select BC cells for dependence on NF-K $\beta$ signaling and thus promote endocrine resistance.

Protein-tyrosine phosphatase 1B (PTP1B) reverts PERK phosphorylation in response to EnR stress (138). PTP1B specifically de-phosphorylates Tyr616, resulting in PERK inactivation and attenuation of this UPR branch (138). PTP1B is commonly over-expressed in BC $(139,140)$, being correlated with ER (141). Therefore, it would be interesting to know if PTP1B is implicated in BC endocrine resistance through downregulation of the pro-apoptotic PERK-CHOP pathway.

PERK and elF2 $\alpha$ were found overexpressed in BC samples and were significantly associated with high histological grade and with tumor-infiltrating lymphocytes (142). However, it is important to consider that most of the performed studies lack information about therapy and the phosphorylation state of these two proteins, being of interest to verify their expression pattern before and after endocrine therapy.

- $\quad$ PERK/elF2 $\alpha$ in prostate cancer: Androgen treatment in LNCaP cells had no short-term effect on total or phosphorylated PERK and elF2 $\alpha$ protein, while it reduced 
their levels after $24 \mathrm{~h}$ treatment and until the end of the time-course experiment at $72 \mathrm{~h}$. Interestingly, ATF4 and CHOP protein levels increased in a time-dependent manner from $24 \mathrm{~h}$ until $72 \mathrm{~h}(61)$. The authors suggest that upon androgen treatment dephosphorylation of PERK and elF2aresults in a general increase in protein synthesis, compensating the effects observed for mRNA levels. However, in this work CHOP increase wasn't sufficient to trigger apoptosis as it was counterbalanced by strong activation of IRE $1 \alpha / X B P-1$ pathway (61). On the other hand, when LNCaP cells were cultured in serum-starvation, a rapid PERK/p-eIF2 $\alpha$ induction occurred after $2 \mathrm{~h}$ androgen treatment and was maintained above basal levels for up to $24 \mathrm{~h}$, while $\mathrm{CHOP}$ protein levels were also found to be upregulated after $24 \mathrm{~h}$ of starvation (93). Taken together, these data are somewhat contradictory as to whether androgens activate or inhibit PERK/elF2 $\alpha$ branch; yet coincide with the observation that $\mathrm{CHOP}$ activation does not lead to increased apoptosis. Currently, there is no information regarding ARmediated anticipatory/non-genomic activation of this arm, neither its regulation by AR antagonists, nor its function on castration-resistance phenotype.

ATF6 arm: ATF6 activation and its association with BC and PC has received much less attention than IRE1 $\alpha$ or PERK. In T47-D BC cells, E2 increased ATF6 proteolysis transiently between 2 and 4 hours after treatment followed by expression of $\mathrm{BiP}$ and other chaperones (66). It is known from studies performed in mice and fibroblasts thatATF6 is activated under mild stress conditions to stimulate XBP-1u transcription and prepare cells for possible IRE1 $\alpha$ branch activation if stress persists $(39,143)$. In line with this, continued activation of ATF6 and IRE1 1 during chronic EnR stress in LNCaP cells and in prostate glands of ETS related gene (ERG) transgenic mouse model due to AR aggregation, induced survival pathways and selective pressure throughout the neoplastic process (60). 


\section{Conclusions and perspectives}

Although the mechanisms by which UPR participates in tumorigenesis and interferes with anti-cancer therapies are not completely clear, current information supports its role in up-regulating pro-survival signaling, being correlated with poor response and resistance to endocrine therapy, disease progression, metastasis, shorter time to recurrence and decreased overall survival in both BC and PC patients. Thus, it is important to understand the contribution of each UPR arm and their downstream proteins, in order to disclose targets to enhance therapy response. With this in mind, we have summarized the current knowledge about UPR activation in endocrine response of breast and prostate cancer (Table 1 and Figure 3).

Current knowledge on UPR function in endocrine response and resistance in PC cells is very limited 1. Future studies to provide new insights about the regulation of these pathways and their role in development of castration-resistance are needed. In addition, the effect of $A R$ and ERa agonists and antagonists at different time points leads to a variety of effects on UPR branch activation. Therefore, studies are needed to understand how the diversity of responses are modulated by acute or chronic exposure to different agonists, antagonists and other endocrine regulators as well as how the mutational landscape of the cell contributes to shaping the UPR outcome. This will further our understanding of how selective UPR arm activation can influence to clonal evolution.

An increasing number of publications support the idea that BiP is a key participant in BC and PC tumorigenesis. Its up-regulation is correlated with decreased apoptosis, promotion of angiogenesis, tumor progression and development of therapy resistance. One possible explanation is that higher BiP levels confer a better proteotoxic resolution and would increase the amount of BiP bound to IRE1a, PERK and ATF6, this in turn would result in a milder UPR activation favoring survival responses rather than cytostasis or death. On the other hand, it was observed that $\mathrm{BiP}$ 
is involved in growth inhibitory mechanisms in endocrine therapy sensitive cancer cells that are lost in resistant cells, the mechanisms behind this remain to be disclosed. However, the fact that BiP subcellular distribution could impact non-genomic signaling directing cells toward apoptosis or survival needs to be explored.

ATF6 remains the less explored branch of UPR and its interaction with IRE1 $\alpha / X B P-1 s$ and PERK arms remain to be disclosed. It will also be interesting to study ATF6 threshold levels involved in differential UPR activation in high or mild stress conditions. These threshold levels may be important in the context of endocrine therapy resistance.

Since resistant $B C$ and $P C$ cases remain a major clinical problem, new therapeutic approaches and better predictors of therapeutic response are clearly needed. Due to UPR involvement in cell proliferation, apoptosis evasion and drugresistance phenotypes targeting these pathways have recently emerged as a promising strategy in anti-cancer therapy. Thus, testing the expression levels of proteins of the UPR, such as BiP or XBP-1as biomarkers in BC and PC may be useful to predict therapy responsiveness and would reduce the exposure to therapeutic agents that are not likely to be beneficial. Additionally, combining existent BC and PC therapies with modulation of UPR network may be a promising strategy to sensitize resistant cancer cells to therapy and to improve clinical outcome.

\section{Acknowledgments}

This work was supported by national funds through FCT - Fundação para a Ciência e a Tecnologia - within the projects UID/BIM/04501/2013 and POCI-01-0145-FEDER007628granted to the Aveiro Institute for Biomedicine. Inês Direito is supported by an FCT PhD fellowship SFRH/BD/123821/2016.

\section{Author contributions}


All the authors made substantial contributions to the conception, design, critical discussion and writing of the manuscript.

\section{Conflict of interest}

Authors declare no conflict of interest. 


\section{References}

1. Ferlay J, S.I., Ervik M, Dikshit R, Eser S, Mathers C, Rebelo M, Parkin DM, Forman D, Bray, F. (2013) GLOBOCAN 2012 v1.0, Cancer Incidence and Mortality Worldwide: IARC CancerBase No. 11 [Internet]. Lyon, France: International Agency for Research on Cancer; 2013. Available from: http://globocan.iarc.fr, accessed on 12/9/2016. International Agency for Research on Cancer, Lyon, France.

2. Clarke, R., et al. (2003) Antiestrogen resistance in breast cancer and the role of estrogen receptor signaling. Oncogene, 22, 7316-39.

3. Musgrove, E.A., et al. (2009) Biological determinants of endocrine resistance in breast cancer. Nat Rev Cancer, 9, 631-43.

4. Schweizer, M.T., et al. (2015) Persistent androgen receptor addiction in castrationresistant prostate cancer. J Hematol Oncol, 8, 128.

5. Arpino, G., et al. (2008) Crosstalk between the estrogen receptor and the HER tyrosine kinase receptor family: molecular mechanism and clinical implications for endocrine therapy resistance. Endocr Rev, 29, 217-33.

6. Giuliano, M., et al. (2013) Bidirectional Crosstalk between the Estrogen Receptor and Human Epidermal Growth Factor Receptor 2 Signaling Pathways in Breast Cancer: Molecular Basis and Clinical Implications. Breast Care (Basel), 8, 256-62.

7. Britton, D.J., et al. (2006) Bidirectional cross talk between ERalpha and EGFR signalling pathways regulates tamoxifen-resistant growth. Breast Cancer Res Treat, 96, 131-46.

8. Osborne, C.K., et al. (2005) Crosstalk between estrogen receptor and growth factor receptor pathways as a cause for endocrine therapy resistance in breast cancer. Clin Cancer Res, 11, 865s-70s.

9. Zhu, M.L., et al. (2008) Androgen receptor and growth factor signaling cross-talk in prostate cancer cells. Endocr Relat Cancer, 15, 841-9.

10. Wang, Y., et al. (2007) Cross-talk between the androgen receptor and the phosphatidylinositol 3-kinase/Akt pathway in prostate cancer. Curr Cancer Drug Targets, 7, 591-604.

11. Jeselsohn, R., et al. (2017) The Evolving Role of the Estrogen Receptor Mutations in Endocrine Therapy-Resistant Breast Cancer. Curr Oncol Rep, 19, 35.

12. Lonergan, P.E., et al. (2011) Androgen receptor signaling in prostate cancer development and progression. J Carcinog, 10, 20.

13. Mao, C., et al. (2016) Antiestrogen Resistant Cell Lines Expressing Estrogen Receptor $\alpha$ Mutations Upregulate the Unfolded Protein Response and are Killed by BHPI. Sci Rep, 6, 34753.

14. Clarke, R., et al. (2015) Unfolding the Role of Stress Response Signaling in Endocrine Resistant Breast Cancers. Front Oncol, 5, 140.

15. Pootrakul, L., et al. (2006) Expression of stress response protein Grp78 is associated with the development of castration-resistant prostate cancer. Clin Cancer Res, 12, 5987-93.

16. Cook, K.L., et al. (2012) Glucose-regulated protein 78 controls cross-talk between apoptosis and autophagy to determine antiestrogen responsiveness. Cancer Res, 72, 3337-49.

17. Labbadia, J., et al. (2015) The biology of proteostasis in aging and disease. Annu Rev Biochem, 84, 435-64.

18. Gomez, B.P., et al. (2007) Human X-box binding protein-1 confers both estrogen independence and antiestrogen resistance in breast cancer cell lines. FASEB J, 21, 4013-27.

19. Parmar, J.H., et al. (2013) Modelling the effect of GRP78 on anti-oestrogen sensitivity and resistance in breast cancer. Interface Focus, 3, 20130012. 
20. Ming, J., et al. (2015) A novel chemical, STF-083010, reverses tamoxifen-related drug resistance in breast cancer by inhibiting IRE1/XBP1. Oncotarget, 6, 40692-703.

21. Xu, H., et al. (2008) Diminished feedback regulation of proteasome expression and resistance to proteasome inhibitors in breast cancer cells. Breast Cancer Res Treat, 107, 267-74.

22. Bursch, W., et al. (1996) Active cell death induced by the anti-estrogens tamoxifen and ICl 164384 in human mammary carcinoma cells (MCF-7) in culture: the role of autophagy. Carcinogenesis, 17, 1595-607.

23. Qadir, M.A., et al. (2008) Macroautophagy inhibition sensitizes tamoxifen-resistant breast cancer cells and enhances mitochondrial depolarization. Breast Cancer Res Treat, 112, 389-403.

24. Rocchi, P., et al. (2004) Heat shock protein 27 increases after androgen ablation and plays a cytoprotective role in hormone-refractory prostate cancer. Cancer Res, 64, 6595-602.

25. Albertelli, M.A., et al. (2008) Glutamine tract length of human androgen receptors affects hormone-dependent and -independent prostate cancer in mice. Hum Mol Genet, 17, 98-110.

26. Vandewynckel, Y.P., et al. (2013) The paradox of the unfolded protein response in cancer. Anticancer Res, 33, 4683-94.

27. Wang, W.A., et al. (2014) Endoplasmic reticulum stress associated responses in cancer. Biochim Biophys Acta, 1843, 2143-9.

28. Clarke, R., et al. (2011) Endoplasmic reticulum stress, the unfolded protein response, and gene network modeling in antiestrogen resistant breast cancer. Horm Mol Biol Clin Investig, 5, 35-44.

29. Yoshida, H., et al. (2006) pXBP1(U) encoded in XBP1 pre-mRNA negatively regulates unfolded protein response activator pXBP1(S) in mammalian ER stress response. J Cell Biol, 172, 565-75.

30. Davies, M.P., et al. (2008) Expression and splicing of the unfolded protein response gene XBP-1 are significantly associated with clinical outcome of endocrine-treated breast cancer. Int J Cancer, 123, 85-8.

31. Hetz, C., et al. (2018) The Unfolded Protein Response and Cell Fate Control. Mol Cell, 69, 169-181.

32. Shi, Y., et al. (1998) Identification and characterization of pancreatic eukaryotic initiation factor 2 alpha-subunit kinase, PEK, involved in translational control. Mol Cell Biol, 18, 7499-509.

33. Holcik, M., et al. (2005) Translational control in stress and apoptosis. Nat Rev Mol Cell Biol, 6, 318-27.

34. Clarke, H.J., et al. (2014) Endoplasmic reticulum stress in malignancy. Cancer Cell, 25, 563-73.

35. Puthalakath, H., et al. (2007) ER stress triggers apoptosis by activating BH3-only protein Bim. Cell, 129, 1337-49.

36. Brush, M.H., et al. (2003) Growth arrest and DNA damage-inducible protein GADD34 targets protein phosphatase 1 alpha to the endoplasmic reticulum and promotes dephosphorylation of the alpha subunit of eukaryotic translation initiation factor 2 . Mol Cell Biol, 23, 1292-303.

37. Clarke, R., et al. (2012) Endoplasmic reticulum stress, the unfolded protein response, autophagy, and the integrated regulation of breast cancer cell fate. Cancer Res, 72, 1321-31.

38. Okada, T., et al. (2002) Distinct roles of activating transcription factor 6 (ATF6) and double-stranded RNA-activated protein kinase-like endoplasmic reticulum kinase 
(PERK) in transcription during the mammalian unfolded protein response. Biochem $J$, 366, 585-94.

39. Wu, J., et al. (2007) ATF6alpha optimizes long-term endoplasmic reticulum function to protect cells from chronic stress. Dev Cell, 13, 351-64.

40. Bluemn, E.G., et al. (2012) The androgen/androgen receptor axis in prostate cancer. Curr Opin Oncol, 24, 251-7.

41. Hasegawa, D., et al. (2015) Epithelial Xbp1 is required for cellular proliferation and differentiation during mammary gland development. Mol Cell Biol, 35, 1543-56.

42. Tsuchiya, M., et al. (2017) The role of unfolded protein response in differentiation of mammary epithelial cells. Biochem Biophys Res Commun, 484, 903-908.

43. Sequeira, S.J., et al. (2007) Inhibition of proliferation by PERK regulates mammary acinar morphogenesis and tumor formation. PLoS One, 2, e615.

44. Bagheri-Yarmand, R., et al. (2003) Activating transcription factor 4 overexpression inhibits proliferation and differentiation of mammary epithelium resulting in impaired lactation and accelerated involution. J Biol Chem, 278, 17421-9.

45. Bobrovnikova-Marjon, E., et al. (2008) PERK-dependent regulation of lipogenesis during mouse mammary gland development and adipocyte differentiation. Proc Natl Acad Sci U S A, 105, 16314-9.

46. Wärri, A., et al. (2018) Autophagy and unfolded protein response (UPR) regulate mammary gland involution by restraining apoptosis-driven irreversible changes. Cell Death Discov, 5, 40.

47. Dória, M.L., et al. (2014) Fatty acid and phospholipid biosynthetic pathways are regulated throughout mammary epithelial cell differentiation and correlate to breast cancer survival. FASEB J, 28, 4247-64.

48. Talukder, A.H., et al. (2002) Expression and transactivating functions of the bZIP transcription factor GADD153 in mammary epithelial cells. Oncogene, 21, 4289-300.

49. Barone, M.V., et al. (1994) CHOP (GADD153) and its oncogenic variant, TLS-CHOP, have opposing effects on the induction of G1/S arrest. Genes Dev, 8, 453-64.

50. Hanahan, D., et al. (2011) Hallmarks of cancer: the next generation. Cell, 144, 646-74.

51. Jahn, T.R., et al. (2008) Folding versus aggregation: polypeptide conformations on competing pathways. Arch Biochem Biophys, 469, 100-17.

52. Carvalhal Marques, F., et al. (2015) The roles of cellular and organismal aging in the development of late-onset maladies. Annu Rev Pathol, 10, 1-23.

53. Chen, L., et al. (2005) Increased proteasome activity, ubiquitin-conjugating enzymes, and EEF1A translation factor detected in breast cancer tissue. Cancer Res, 65, 5599606.

54. Della Donna, L., et al. (2012) Radioresistance of prostate cancer cells with low proteasome activity. Prostate, 72, 868-74.

55. Vlashi, E., et al. (2013) Targeted elimination of breast cancer cells with low proteasome activity is sufficient for tumor regression. Breast Cancer Res Treat, 141, 197-203.

56. Stacer, A.C., et al. (2015) Imaging Reporters for Proteasome Activity Identify Tumorand Metastasis-Initiating Cells. Mol Imaging, 14, 414-28.

57. Banno, A., et al. (2016) Downregulation of $26 \mathrm{~S}$ proteasome catalytic activity promotes epithelial-mesenchymal transition. Oncotarget, 7, 21527-41.

58. Bartkowiak, K., et al. (2015) Disseminated Tumor Cells Persist in the Bone Marrow of Breast Cancer Patients through Sustained Activation of the Unfolded Protein Response. Cancer Res, 75, 5367-77.

59. Thorpe, J.A., et al. (2010) IRE1alpha controls cyclin A1 expression and promotes cell proliferation through XBP-1. Cell Stress Chaperones, 15, 497-508. 
60. Sreenath, T.L., et al. (2017) ETS Related Gene mediated Androgen Receptor Aggregation and Endoplasmic Reticulum Stress in Prostate Cancer Development. Sci Rep, 7, 1109.

61. Sheng, X., et al. (2015) Divergent androgen regulation of unfolded protein response pathways drives prostate cancer. EMBO Mol Med, 7, 788-801.

62. Liu, J., et al. (2017) Activation of UPR Signaling Pathway is Associated With the Malignant Progression and Poor Prognosis in Prostate Cancer. Prostate, 77, 274-281.

63. Kobylewski, S.E., et al. (2017) Activation of the EIF2 $\alpha /$ ATF4 and ATF6 Pathways in DU145 Cells by Boric Acid at the Concentration Reported in Men at the US Mean Boron Intake. Biol Trace Elem Res, 176, 278-293.

64. Daneshmand, S., et al. (2007) Glucose-regulated protein GRP78 is up-regulated in prostate cancer and correlates with recurrence and survival. Hum Pathol, 38, 1547-52.

65. Arap, M.A., et al. (2004) Cell surface expression of the stress response chaperone GRP78 enables tumor targeting by circulating ligands. Cancer Cell, 6, 275-84.

66. Andruska, N., et al. (2015) Anticipatory estrogen activation of the unfolded protein response is linked to cell proliferation and poor survival in estrogen receptor $\alpha$-positive breast cancer. Oncogene, 34, 3760-9.

67. Nami, B., et al. (2016) Tunicamycin-induced endoplasmic reticulum stress reduces in vitro subpopulation and invasion of CD44+/CD24- phenotype breast cancer stem cells. Exp Toxicol Pathol, 68, 419-26.

68. So, A.Y., et al. (2009) The unfolded protein response during prostate cancer development. Cancer Metastasis Rev, 28, 219-23.

69. Heldring, N., et al. (2007) Estrogen receptors: how do they signal and what are their targets. Physiol Rev, 87, 905-31.

70. Marino, M., et al. (2006) Estrogen signaling multiple pathways to impact gene transcription. Curr Genomics, 7, 497-508.

71. Kato, S., et al. (1995) Activation of the estrogen receptor through phosphorylation by mitogen-activated protein kinase. Science, 270, 1491-4.

72. Gazit, G., et al. (1999) De-regulation of GRP stress protein expression in human breast cancer cell lines. Breast Cancer Res Treat, 54, 135-46.

73. Cornford, P.A., et al. (2000) Heat shock protein expression independently predicts clinical outcome in prostate cancer. Cancer Res, 60, 7099-105.

74. Liberek, K., et al. (2008) Chaperones in control of protein disaggregation. EMBO J, 27, 328-35.

75. Wang, D.Y., et al. (2004) Identification of estrogen-responsive genes by complementary deoxyribonucleic acid microarray and characterization of a novel early estrogen-induced gene: EEIG1. Mol Endocrinol, 18, 402-11.

76. Andruska, N.D., et al. (2015) Estrogen receptor $\alpha$ inhibitor activates the unfolded protein response, blocks protein synthesis, and induces tumor regression. Proc Natl Acad Sci U S A, 112, 4737-42.

77. Dhamad, A.E., et al. (2016) Systematic Proteomic Identification of the Heat Shock Proteins (Hsp) that Interact with Estrogen Receptor Alpha (ER $\alpha)$ and Biochemical Characterization of the ER $\alpha$-Hsp70 Interaction. PLoS One, 11, e0160312.

78. Scriven, P., et al. (2009) Activation and clinical significance of the unfolded protein response in breast cancer. Br J Cancer, 101, 1692-8.

79. Ariazi, E.A., et al. (2011) Estrogen induces apoptosis in estrogen deprivation-resistant breast cancer through stress responses as identified by global gene expression across time. Proc Natl Acad Sci U S A, 108, 18879-86. 
80. Fu, Y., et al. (2007) GRP78/BiP inhibits endoplasmic reticulum BIK and protects human breast cancer cells against estrogen starvation-induced apoptosis. Cancer Res, 67, 3734-40.

81. Reddy, R.K., et al. (2003) Endoplasmic reticulum chaperone protein GRP78 protects cells from apoptosis induced by topoisomerase inhibitors: role of ATP binding site in suppression of caspase-7 activation. J Biol Chem, 278, 20915-24.

82. Rao, R.V., et al. (2002) Coupling endoplasmic reticulum stress to the cell death program: role of the ER chaperone GRP78. FEBS Lett, 514, 122-8.

83. Ermakova, S.P., et al. (2006) (-)-Epigallocatechin gallate overcomes resistance to etoposide-induced cell death by targeting the molecular chaperone glucose-regulated protein 78. Cancer Res, 66, 9260-9.

84. Cook, K.L., et al. (2016) Endoplasmic Reticulum Stress Protein GRP78 Modulates Lipid Metabolism to Control Drug Sensitivity and Antitumor Immunity in Breast Cancer. Cancer Res, 76, 5657-5670.

85. Basseri, S., et al. (2012) Endoplasmic reticulum stress and lipid metabolism: mechanisms and therapeutic potential. Biochem Res Int, 2012, 841362.

86. Shajahan-Haq, A.N., et al. (2014) MYC regulates the unfolded protein response and glucose and glutamine uptake in endocrine resistant breast cancer. Mol Cancer, 13, 239.

87. Shajahan-Haq, A.N., et al. (2017) EGR1 regulates cellular metabolism and survival in endocrine resistant breast cancer. Oncotarget, 8, 96865-96884.

88. Fernandez, P.M., et al. (2000) Overexpression of the glucose-regulated stress gene GRP78 in malignant but not benign human breast lesions. Breast Cancer Res Treat, 59, 15-26.

89. Raiter, A., et al. (2014) Pharmacological induction of cell surface GRP78 contributes to apoptosis in triple negative breast cancer cells. Oncotarget, 5, 11452-63.

90. Yerushalmi, R., et al. (2015) Cell surface GRP78: A potential marker of good prognosis and response to chemotherapy in breast cancer. Oncol Lett, 10, 2149-2155.

91. Jakobsen, C.G., et al. (2007) Phage display derived human monoclonal antibodies isolated by binding to the surface of live primary breast cancer cells recognize GRP78. Cancer Res, 67, 9507-17.

92. Mintz, P.J., et al. (2003) Fingerprinting the circulating repertoire of antibodies from cancer patients. Nat Biotechnol, 21, 57-63.

93. Bennett, H.L., et al. (2010) Androgens modulate autophagy and cell death via regulation of the endoplasmic reticulum chaperone glucose-regulated protein $78 / \mathrm{BiP}$ in prostate cancer cells. Cell Death Dis, 1, e72.

94. Misra, U.K., et al. (2006) Activation and cross-talk between Akt, NF-kappaB, and unfolded protein response signaling in 1-LN prostate cancer cells consequent to ligation of cell surface-associated GRP78. J Biol Chem, 281, 13694-707.

95. Fu, Y., et al. (2008) Pten null prostate tumorigenesis and AKT activation are blocked by targeted knockout of ER chaperone GRP78/BiP in prostate epithelium. Proc Natl Acad Sci U S A, 105, 19444-9.

96. Zhang, Y., et al. (2013) Cancer cells resistant to therapy promote cell surface relocalization of GRP78 which complexes with PI3K and enhances PI(3,4,5)P3 production. PLoS One, 8, e80071.

97. Burikhanov, R., et al. (2009) The tumor suppressor Par-4 activates an extrinsic pathway for apoptosis. Cell, 138, 377-88.

98. Lee, A.S. (2009) The Par-4-GRP78 TRAIL, more twists and turns. Cancer Biol Ther, 8, 2103-5. 
99. Misra, U.K., et al. (2005) The role of MTJ-1 in cell surface translocation of GRP78, a receptor for alpha 2-macroglobulin-dependent signaling. J Immunol, 174, 2092-7.

100. Zhang, Y., et al. (2010) Cell surface relocalization of the endoplasmic reticulum chaperone and unfolded protein response regulator GRP78/BiP. J Biol Chem, 285, 15065-75.

101. Ding, L., et al. (2003) Ligand-independent activation of estrogen receptor alpha by XBP1. Nucleic Acids Res, 31, 5266-74.

102. Milani, M., et al. (2009) The role of ATF4 stabilization and autophagy in resistance of breast cancer cells treated with Bortezomib. Cancer Res, 69, 4415-23.

103. Hu, R., et al. (2015) NF-KB signaling is required for XBP1 (unspliced and spliced)mediated effects on antiestrogen responsiveness and cell fate decisions in breast cancer. Mol Cell Biol, 35, 379-90.

104. Romero-Ramirez, L., et al. (2004) XBP1 is essential for survival under hypoxic conditions and is required for tumor growth. Cancer Res, 64, 5943-7.

105. Chen, X., et al. (2014) XBP1 promotes triple-negative breast cancer by controlling the HIF1 a pathway. Nature, 508, 103-7.

106. Yang, J., et al. (2015) Estrogen receptor- $\alpha$ directly regulates the hypoxia-inducible factor 1 pathway associated with antiestrogen response in breast cancer. Proc Natl Acad Sci U S A, 112, 15172-7.

107. Sas, L., et al. (2012) The interaction between ER and NFKB in resistance to endocrine therapy. Breast Cancer Res, 14, 212.

108. Nakshatri, H., et al. (1997) Constitutive activation of NF-kappaB during progression of breast cancer to hormone-independent growth. Mol Cell Biol, 17, 3629-39.

109. Tam, A.B., et al. (2012) ER stress activates NF-KB by integrating functions of basal IKK activity, IRE1 and PERK. PLoS One, 7, e45078.

110. Cotrim, C.Z., et al. (2013) Estrogen receptor beta growth-inhibitory effects are repressed through activation of MAPK and PI3K signalling in mammary epithelial and breast cancer cells. Oncogene, 32, 2390-402.

111. Thomas, C., et al. (2011) The different roles of ER subtypes in cancer biology and therapy. Nat Rev Cancer, 11, 597-608.

112. Lindberg, K., et al. (2010) Expression of estrogen receptor beta increases integrin alpha1 and integrin beta1 levels and enhances adhesion of breast cancer cells. J Cell Physiol, 222, 156-67.

113. Lindberg, K., et al. (2011) Estrogen receptor $\beta$ represses Akt signaling in breast cancer cells via downregulation of HER2/HER3 and upregulation of PTEN: implications for tamoxifen sensitivity. Breast Cancer Res, 13, R43.

114. Rajapaksa, G., et al. (2016) Estrogen signaling and unfolded protein response in breast cancer. J Steroid Biochem Mol Biol, 163, 45-50.

115. Rajapaksa, G., et al. (2015) ERß decreases breast cancer cell survival by regulating the IRE1/XBP-1 pathway. Oncogene, 34, 4130-41.

116. Zhu, Y., et al. (2006) Expression patterns among interferon regulatory factor-1, human $\mathrm{X}$-box binding protein-1, nuclear factor kappa B, nucleophosmin, estrogen receptoralpha and progesterone receptor proteins in breast cancer tissue microarrays. Int $J$ Oncol, 28, 67-76.

117. Fujimoto, T., et al. (2003) Upregulation and overexpression of human X-box binding protein 1 (hXBP-1) gene in primary breast cancers. Breast Cancer, 10, 301-6.

118. Murphy, L.C., et al. (2012) The role of estrogen receptor- $\beta$ in breast cancer. Semin Reprod Med, 30, 5-13. 
119. Ishii, Y., et al. (2011) Bortezomib enhances the efficacy of fulvestrant by amplifying the aggregation of the estrogen receptor, which leads to a proapoptotic unfolded protein response. Clin Cancer Res, 17, 2292-300.

120. Beelen, K., et al. (2014) PI3K/AKT/mTOR pathway activation in primary and corresponding metastatic breast tumors after adjuvant endocrine therapy. Int $J$ Cancer, 135, 1257-63.

121. Paplomata, E., et al. (2014) The PI3K/AKT/mTOR pathway in breast cancer: targets, trials and biomarkers. Ther Adv Med Oncol, 6, 154-66.

122. Tokunaga, E., et al. (2006) Activation of PI3K/Akt signaling and hormone resistance in breast cancer. Breast Cancer, 13, 137-44.

123. Ciruelos Gil, E.M. (2014) Targeting the PI3K/AKT/mTOR pathway in estrogen receptorpositive breast cancer. Cancer Treat Rev, 40, 862-71.

124. deGraffenried, L.A., et al. (2004) Inhibition of mTOR activity restores tamoxifen response in breast cancer cells with aberrant Akt Activity. Clin Cancer Res, 10, 8059-67.

125. Mounir, Z., et al. (2011) Akt determines cell fate through inhibition of the PERK-elF2 $\alpha$ phosphorylation pathway. Sci Signal, 4, ra62.

126. Bobrovnikova-Marjon, E., et al. (2010) PERK promotes cancer cell proliferation and tumor growth by limiting oxidative DNA damage. Oncogene, 29, 3881-95.

127. Nogueira, V., et al. (2008) Akt determines replicative senescence and oxidative or oncogenic premature senescence and sensitizes cells to oxidative apoptosis. Cancer Cell, 14, 458-70.

128. Cullinan, S.B., et al. (2003) Nrf2 is a direct PERK substrate and effector of PERKdependent cell survival. Mol Cell Biol, 23, 7198-209.

129. Cullinan, S.B., et al. (2004) PERK-dependent activation of Nrf2 contributes to redox homeostasis and cell survival following endoplasmic reticulum stress. J Biol Chem, 279, 20108-17.

130. Del Vecchio, C.A., et al. (2014) De-differentiation confers multidrug resistance via noncanonical PERK-Nrf2 signaling. PLoS Biol, 12, e1001945.

131. Szegezdi, E., et al. (2006) Mediators of endoplasmic reticulum stress-induced apoptosis. EMBO Rep, 7, 880-5.

132. Zhou, Y., et al. (2005) The NFkappaB pathway and endocrine-resistant breast cancer. Endocr Relat Cancer, 12 Suppl 1, S37-46.

133. Riggins, R.B., et al. (2005) The nuclear factor kappa B inhibitor parthenolide restores ICI 182,780 (Faslodex; fulvestrant)-induced apoptosis in antiestrogen-resistant breast cancer cells. Mol Cancer Ther, 4, 33-41.

134. Gu, Z., et al. (2002) Association of interferon regulatory factor-1, nucleophosmin, nuclear factor-kappaB, and cyclic AMP response element binding with acquired resistance to Faslodex (ICI 182,780). Cancer Res, 62, 3428-37.

135. Nozaki, S., et al. (2001) Repression of GADD153/CHOP by NF-kappaB: a possible cellular defense against endoplasmic reticulum stress-induced cell death. Oncogene, 20, 2178-85.

136. Deng, J., et al. (2004) Translational repression mediates activation of nuclear factor kappa B by phosphorylated translation initiation factor 2. Mol Cell Biol, 24, 10161-8.

137. Chaudhari, N., et al. (2014) A molecular web: endoplasmic reticulum stress, inflammation, and oxidative stress. Front Cell Neurosci, 8, 213.

138. Krishnan, N., et al. (2011) H2S-Induced sulfhydration of the phosphatase PTP1B and its role in the endoplasmic reticulum stress response. Sci Signal, 4, ra86.

139. Wiener, J.R., et al. (1994) Overexpression of the protein tyrosine phosphatase PTP1B in human breast cancer: association with p185c-erbB-2 protein expression. J Natl Cancer Inst, 86, 372-8. 
140. Zhai, Y.F., et al. (1993) Increased expression of specific protein tyrosine phosphatases in human breast epithelial cells neoplastically transformed by the neu oncogene. Cancer Res, 53, 2272-8.

141. Liao, S.C., et al. (2017) Protein tyrosine phosphatase 1B expression contributes to the development of breast cancer. J Zhejiang Univ Sci B, 18, 334-342.

142. Kim, J.Y., et al. (2016) Activation of the PERK-elF2 $\alpha$ Pathway Is Associated with Tumorinfiltrating Lymphocytes in HER2-Positive Breast Cancer. Anticancer Res, 36, 2705-11.

143. Yamamoto, K., et al. (2007) Transcriptional induction of mammalian ER quality control proteins is mediated by single or combined action of ATF6alpha and XBP1. Dev Cell, 13, 365-76. 
Fig.1- Unfolded protein response (UPR) pathways. (A) The UPR is composed of three different effector branches, protein kinase RNA-like endoplasmic reticulum kinase (PERK), inositol-requiring enzyme $1 \alpha(\mathrm{IRE} 1 \alpha)$ and activating transcription factor 6 (ATF6), which in unstressed conditions remain inactive by association with the EnR stressor sensor-binding immunoglobulin protein (BiP); $(\mathrm{B})$ Initiation of UPR is an attempt to restore proteostasis in response to EnR stress provoked by metabolic changes, hypoxia, acidosis, nutrient deprivation and/or gene mutations. The three different UPR effectors are activated by dissociation from the complexes formed with BiP, leaving BiP free to interact with misfolded proteins within the EnR lumen. Activated PERK phosphorylates and inhibits the eukaryotic initiation factor $2 \alpha$ (elF2 $\alpha)$, which turns off protein synthesis. PERK also increases expression of activating transcription factor 4 (ATF4), which in turn induces the expression of anti-oxidative enzymes, promotes amino acid synthesis and protein folding. Dissociation from BiP leads to IRE1a homodimerization and autophosphorylation, activating its endonuclease activity and promoting the subsequent splicing of X-box binding protein 1 (XBP-1) mRNA. Spliced XBP-1 (XBP-1s) gives origin to a transcription factor that up-regulates genes involved in protein folding, lipid metabolism, quality control and endoplasmic-reticulumassociated degradation (ERAD). Finally, upon its release from BiP, ATF6 translocates to the Golgi where it is cleaved by proteases, releasing an $\mathrm{N}$-terminal fragment that acts as a transcription factor to stimulate the transcription of XBP-1u and ERADassociated genes. 
Fig. 2 - Molecular mechanisms of gene expression regulation mediated by Estrogen Receptor $\alpha(E R \alpha)$ and Androgen Receptor (AR). ERa and AR are transcription factors which exert their molecular functions by regulating gene expression. In the genomic pathway, ERa and AR, illustrated here as nuclear factors (NF) bind their agonist, 17ß-estradiol (E2) and dihydrotestosterone (DHT), respectively which leads to their dimerization. Nuclear translocation of receptor-ligand complexes results in 1) direct DNA binding along with coactivators (CA) to form a transcription complex; 2) indirect DNA binding by recruitment of other transcription factors (TF) like Fos/Jun or SP-1. In addition, ERa and AR can be activated by phosphorylation through other signaling pathways, like growth factor signaling, that results in their dimerization, DNA binding and gene regulation in a ligand-independent way. The genomic pathway induces the transcription of genes involved in cell proliferation and metabolism. In the non-genomic pathway ligand binding activates membrane-associated receptors or receptors located in the cytoplasm, promoting the activation of signaling cascades such as phospholipase C (PLC)/protein kinase C (PKCs), Ras/Raf/MAPK, phosphatidyl inositol 3 kinase (PI3K)/Akt and CAMP/ protein kinase A (PKA). The non-genomic pathway results in rapid physiological responses without direct gene regulation by NRs. ERa and AR actions may be blocked by endocrine therapy. Selective receptor modulators such tamoxifen (or its active metabolite 4-hydroxytamoxifen) or Flutamide promote ERa or AR binding, respectively, to DNA and recruit transcriptional corepressors $(\mathrm{CR})$ to inhibit gene expression. Selective receptor disruptors such as Fulvestrant or Bicalutamide, induce ERa or AR proteasomal degradation, respectively. 
Fig.3- Estrogens and anti-estrogens promote UPR activation in breast cells. (A) Upon estrogen treatment all the 3 branches of the UPR are activated preparing the cells for high protein synthesis demands that precedes cell proliferation. PERK, through elf2 $\alpha$ blocks translation and ATF6 and IRE1 $\alpha$ branches are responsible for the induction of molecular chaperones to increase protein folding capacity and induction of endoplasmic-reticulum-associated degradation (ERAD) mechanisms that alleviate endoplasmic reticulum (EnR) overload. (B) In BC cells sensitive to endocrine therapy, $\mathrm{BiP}$ is upregulated in an attempt to resolve EnR stress caused by treatment, IRE1a/ $\mathrm{XBP} 1$ branch is downregulated and in parallel PERK pathway trough elf2 $\alpha$ / ATF4 /CHOP and JNK signaling are upregulated promoting the expression of pro-apoptotic proteins. mTOR signaling seems also to be slightly induced by treatment promoting autophagy that in high levels is also responsible for cell death. (C) Antiestrogen resistant $B C$ cells in response to endocrine therapy activate UPR through BiP /IRE1 $\alpha$ / XBP-1 branch upregulation, ultimately promoting cell survival and antiestrogen resistance by increasing protein folding capacity and activation of ERAD mechanisms. In parallel with BiP, GRP94 is one of the EnR chaperones that is mostly upregulated upon therapy. Simultaneously, PERK through phosphorylation of elf2 $\alpha$ and blocking

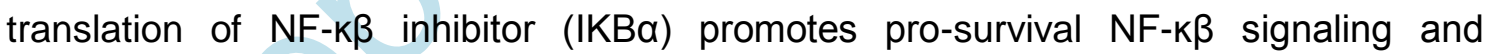
inhibition of pro-apoptotic CHOP. PERK activation also induces NRF-2 anti-oxidant transcription factor contributing for an anti-oxidative stress response. A pro-survival upregulation of a mTOR-independent autophagy program seems also to be activated contributing for cell survival. 
Figure 1
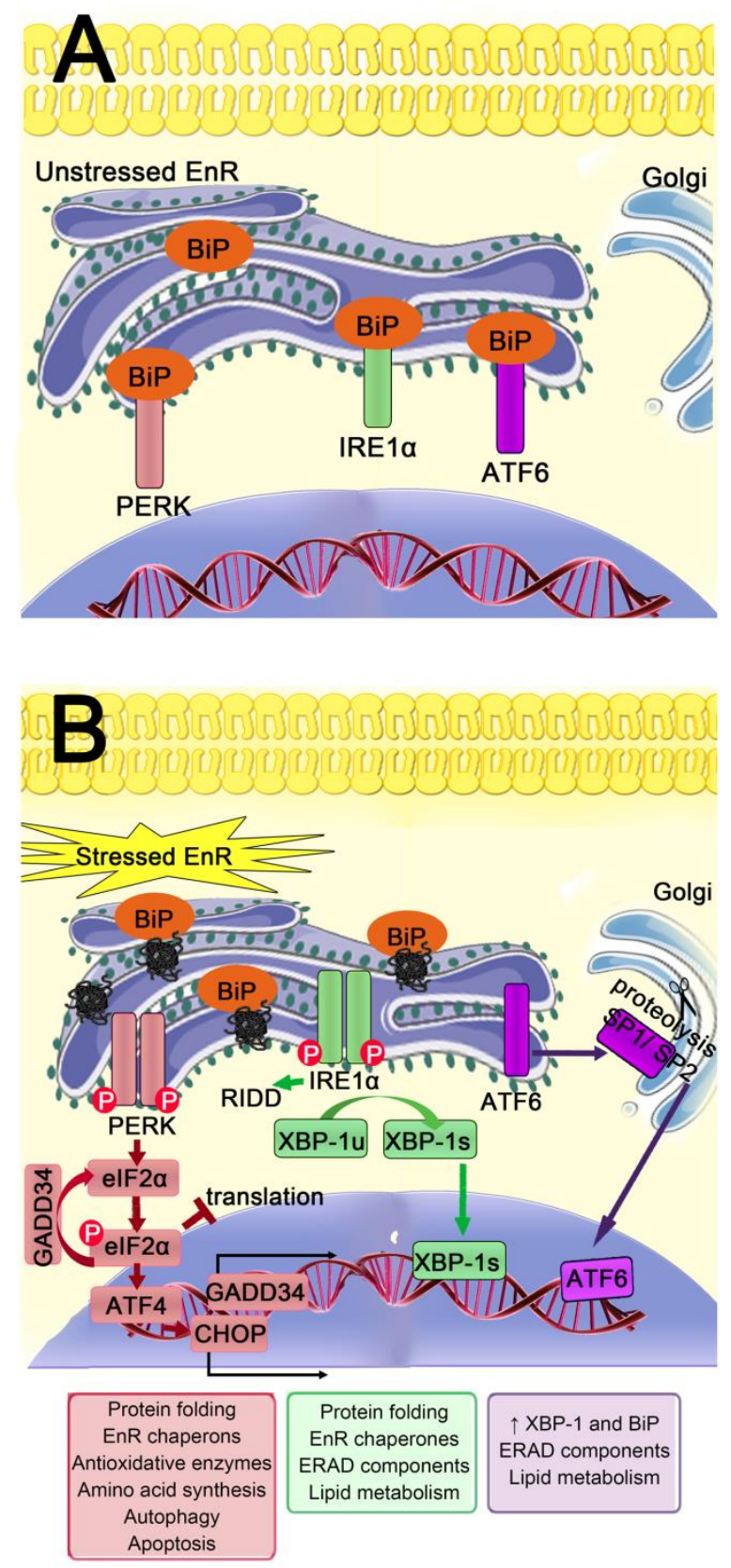

Inês $\mathrm{D}$, et al 
Figure 2

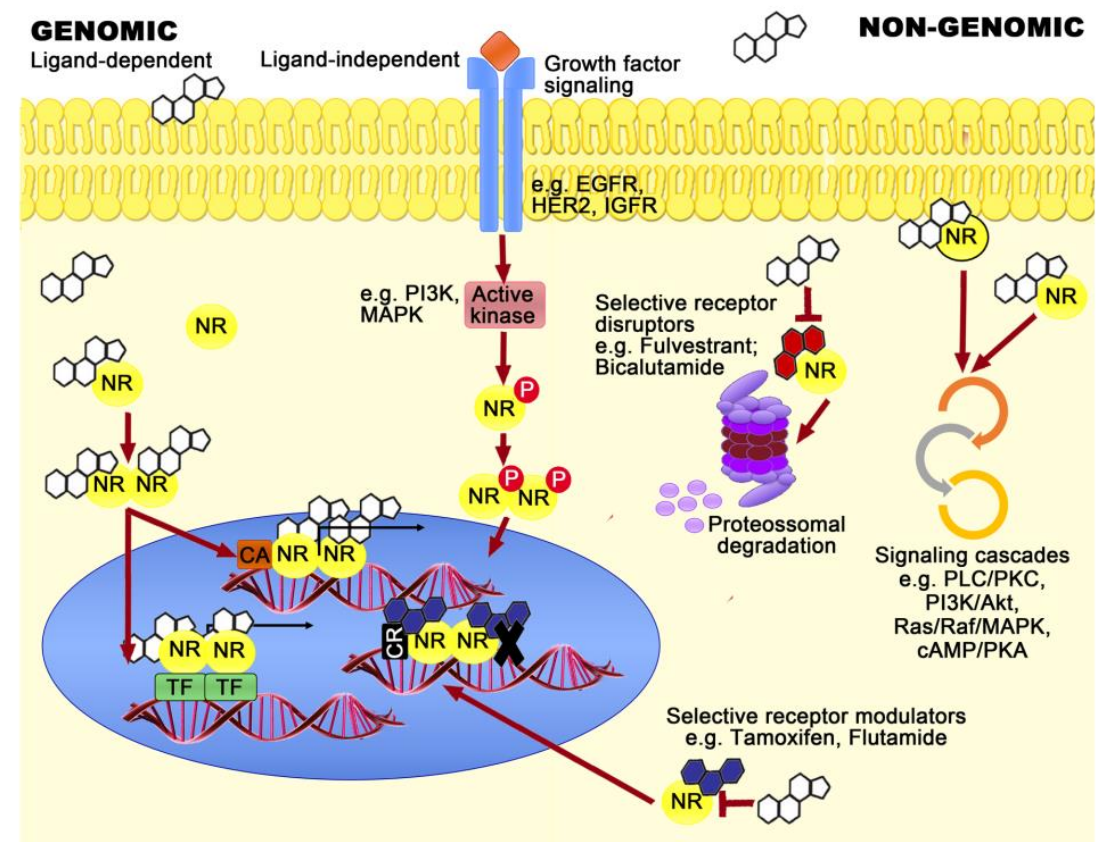

Inês D, et al 
Figure 3

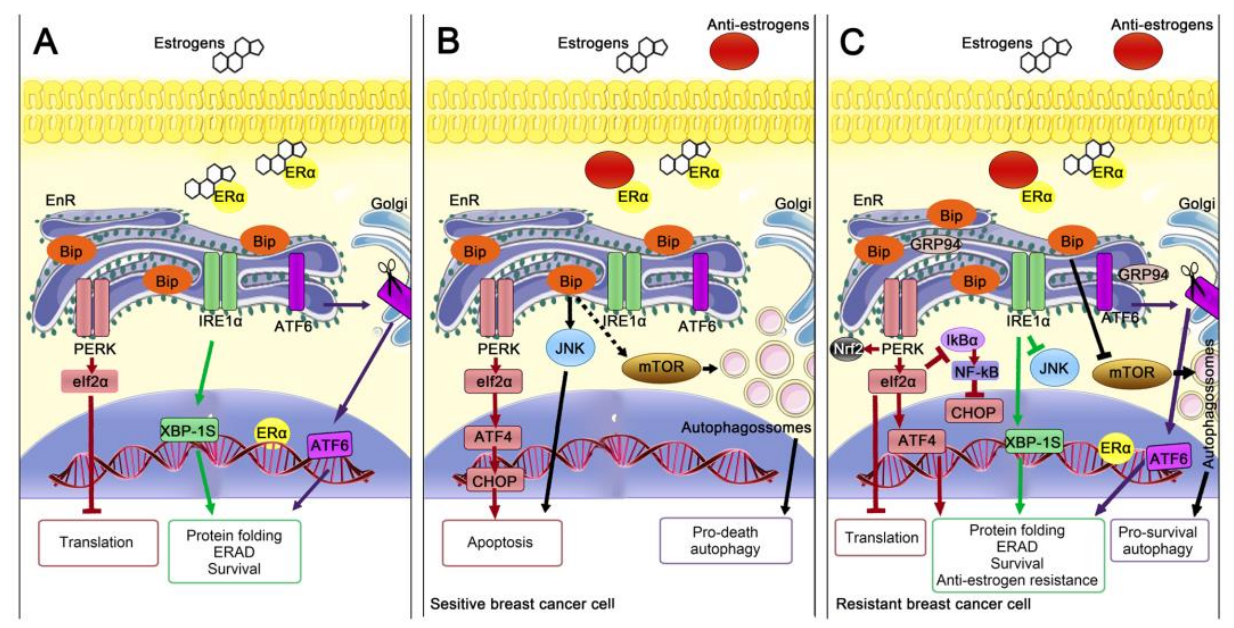

Inês $\mathrm{D}$, et al 
Table 1. Influence of endocrine treatment on UPR activation in Breast and Prostate Cancer cells

\begin{tabular}{|c|c|c|c|c|c|}
\hline $\begin{array}{l}\text { UPR } \\
\text { Branch }\end{array}$ & Condition & Sample & mRNA & Protein & References \\
\hline & & BreastCancer & & & \\
\hline \multirow{3}{*}{ BiP /GRP94 } & EstrogenTreatment & T47D cells, MCF7 cells, MCF7 xenografts & BiP; GRP94 & BiP; GRP94 & $(65,77)$ \\
\hline & $\mathrm{ICI}$ or TAM treatment & $\begin{array}{l}\text { LCC9 cells, LCC } 1 / B i P+\text { cells, T47D cells, LCC1 } \\
\text { cells, MCF7RR cells, TR5 cells }\end{array}$ & $\mathrm{BiP}$ & $\mathrm{BiP}$ & $(19,77)$ \\
\hline & Cancervs Normal & Non-treated BC tissue samples from patients & $\mathrm{BiP}$ & BiP; GRP94 & $(16,65,77,83)$ \\
\hline \multirow{3}{*}{ IRE1a } & EstrogenTreatment & T47D cells, MCF7 cells, MCF7 xenografts & 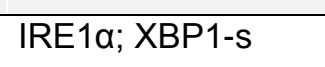 & IRE1a; XBP1-s; XBP1-u & \\
\hline & $\mathrm{ICI}$ or TAM treatment & T47D cells, LCC1 cells & IRE1a; XBP1-s & XBP1-s & (65) \\
\hline & Cancervs Normal & $\begin{array}{l}\text { Invasive ductal adenocarcinoma tissue samples of } \\
\text { patients treated with TAM; Non-treated BC tissue } \\
\text { samples from patients }\end{array}$ & & IRE1a; XBP1 & $(65,77,116)$ \\
\hline \multirow{4}{*}{ PERK } & EstrogenTreatment & T47D cells, MCF7 cells, MCF7 xenografts & PERK; eif2a; ATF4 & PERK; ATF4 & (65) \\
\hline & $\mathrm{ICI}$ or TAM treatment & $\begin{array}{l}\text { LCC9 cells, LCC1/BiP+ cells, MCF7RR cells, TR5 } \\
\text { cells, }\end{array}$ & & PERK; CHOP & $(16,19)$ \\
\hline & & T47D cells, LCC1 cells & & PERK; eif2 $\alpha$; CHOP & $(19,65)$ \\
\hline & Cancervs Normal & $\begin{array}{l}\text { Invasive ductal adenocarcinoma tissue samples of } \\
\text { patients treated with TAM }\end{array}$ & & eif2 $\alpha$ & (65) \\
\hline$\overline{\text { ATF6 }}$ & EstrogenTreatment & T47D cells, MCF7 cells, MCF7 xenografts & ATF6 & & (65) \\
\hline
\end{tabular}




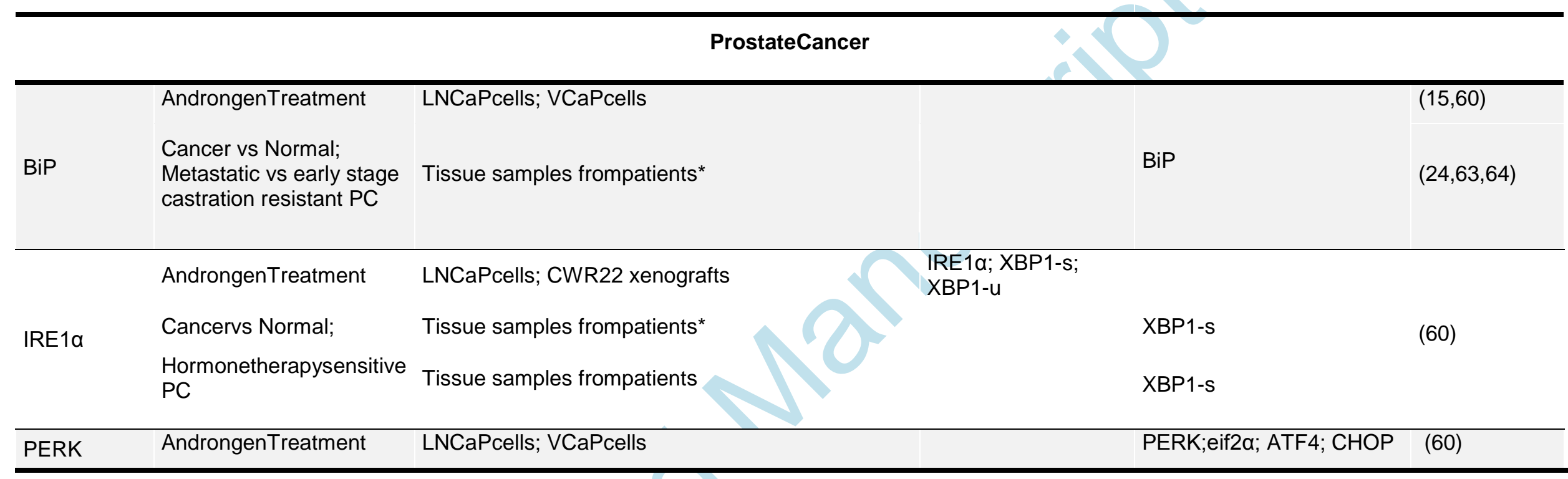

Upregulation in red; downregulation in green. *Without information about patients' treatment. ATF4, activating transcription factor 4; ATF6, activating transcription factor 6; $\mathrm{BC}$, breast cancer; $\mathrm{BiP}$, sensor-binding immunoglobulin protein; CHOP, C/EBP homologous protein; elF2a, eukaryotic initiation factor $2 \alpha$; IRE1 $\alpha$, inositol-requiring enzyme $1 \alpha$; PC, prostate cancer; PERK, protein kinase RNA-like endoplasmic reticulum kinase; TAM, hydroxytamoxifen; XBP-1, X-box binding protein 1; XBP-1s, spliced XBP-1; XBP-1u, unspliced XBP-1. 